\title{
Programa de atención legal para proteger a las víctimas de violencia sexual en el complejo judicial florida norte, Guayaquil. 2020
}

\author{
Mercy Cristina Soledispa Campos \\ soledicampos@hotmail.com \\ Escuela de posgrado: Programa Académico \\ Doctorado en Gestión Pública y Gobernabilidad \\ Universidad Cesar Vallejo \\ Piura - Perú
}

\section{RESUMEN}

El presente trabajo de investigación abordó el tema referido a las víctimas de violencia sexual atendidas en el Complejo Judicial Florida Norte (CJFN), y como se evidencia el trauma psicológico en el que viven, este trabajo tuvo como objetivo proponer un Programa de Atención Legal para proteger a las víctimas de violencia sexual en el CJFN. La metodología tuvo un propósito como Aplicado, enfoque mixto y un alcance Descriptivo y Explicativo, el diseño fue no experimental de corte transversal, se obtuvo una muestra de 137 personas, abogados y personal técnico del CJFN vinculados a temas de violencia sexual. Los resultados mostraron que las directrices legales no dan soporte al programa de atención o asesoría legal, respecto a la atención terapéutica no viene ayudando psicológicamente a la población víctima de violencias, así como de una reducción de riesgos como consecuencia de aplicar una cultura preventivas. Se concluye que una propuesta sobre la implementación de un Programa de Atención Legal protegería a las Víctimas de Violencia Sexual en el CJFN, y que, de acuerdo con la opinión de especialistas sobre esta propuesta, arrojaron un Coeficiente de validez de contenido es 0.916 por lo que se recomienda la pertinencia de implementar y aplicar el programa.

Palabras Clave: programa de atención; legal; victimas; violencia sexual; traumas. 


\title{
Legal assistance program to protect victims of sexual violence in the florida norte judicial complex, Guayaquil. 2020
}

\begin{abstract}
The present research work addressed the issue referred to the victims of sexual violence treated in the Florida North Judicial Complex (CJFN), and as evidenced by the psychological trauma in which they live, this work aimed to propose a Legal Attention Program for protect victims of sexual violence in the CJFN. The methodology had a purpose such as Applied, mixed approach and a Descriptive and Explanatory scope, the design was non-experimental, cross-sectional, a sample of 137 people, lawyers and technical personnel of the CJFN linked to issues of sexual violence was obtained. The results showed that the legal guidelines do not support the program of care or legal advice, with respect to therapeutic care it has not been psychologically helping the population victim of violence, as well as a reduction of risks as a consequence of applying a preventive culture. It is concluded that a proposal on the implementation of a Legal Attention Program would protect Victims of Sexual Violence in the CJFN, and that, according to the opinion of specialists on this proposal, they yielded a content validity coefficient of 0.916 , so that the relevance of implementing and applying the program is recommended.
\end{abstract}

KeyWords: care program; legal; victims; sexual violence; trauma

Artículo recibido: 10 Agosto. 2021 Aceptado para publicación: 07. Setiembre. 2021 Correspondencia: soledicampos@ hotmail.com Conflictos de Interés: Ninguna que declarar 


\section{INTRODUCCIÓN}

Cavagnoud (2018) refiere que las Naciones Unidas conceptualiza la violencia contra las mujeres como toda acción que atente contra la vida y el honor de las personas de sexo femenino como consecuencia de un abuso daño sexual, maltrato físico o psicológico en las mujeres, así mismo las intimidaciones, la privación arbitraria de la libertad, la coacción (ONU, 1993: p. 3). Los actos de la violencia que sufren las mujeres han sido duramente mediatizados a nivel del mundo desde octubre de 2017, esto involucra retos familiares, de cada individuo y políticas con componentes de protección social y de salud pública. El estudio respecto a este tipo de violencia plantea dos formas de mencionarla. La primera se basa en las relaciones de control y poder. La segunda forma se le llama "violencia situacional", la misma que es el resultado de un conflicto abierto entre la pareja, siendo que una disputa acaba en una acción de violencia física más circunstancial (Johnson \& Leone, 2005). (párr. 1-4)

En su revista ACNUR/L (2019), nos dice que la violencia de género y sexual, está representada por actos diversos que va en oposición a la voluntad de una persona y se origina en reglas de género y relaciones que no tienen una igualdad de poder, que incluyen la coerción y amenazas de violencia. (párr. 1)

Giammattei A. (2019) En todo tiempo hemos observado que los impactos que se ocasionan dependen principalmente del tipo de abuso y el contexto en que se ocasiona. Estudios proyectan incrementos de víctimas de abuso sexual o violencia quedan con secuelas, de las cuales se pueden mencionar: miedo, desconfianza, hostilidad a el sexo que lo agredió o hacia su familia, ansiedad, vergüenza, huida de casa, culpa, bajo rendimiento escolar, consumo de drogas, elevada insatisfacción y disfunción sexual, angustia, depresión. Los efectos se incrementan, en tanto más cercano sentimentalmente sea quien le causo la agresión, mientras mayor haya sido el tiempo de lo sucedido, mientras más violencia se haya utilizado y menos credibilidad se brindó a la víctima.

Holt K. (2020), en su reportaje menciona que, en función a muchos informes de violaciones horribles, acontecidas en diferentes lugares del mundo en las últimas semanas, como Bangladesh, Marruecos, India, Argelia, Pakistán, Nigeria y Tunez, entre otros, la población se ha indignado de manera justificada y ha solicitado justicia para las víctimas, así como adoptar las medidas necesarias para prevenir las agresiones sexuales, enfatizó Michelle Bachelet, quien compartió el enfado de las personas afectadas y se 
solidarizó con las víctimas, pero de igual manera hizo saber su intranquilidad ante los invocaciones y la aprobación de leyes que proponen aplicar penas inhumanas y crueles, diferente de la pena de muerte, para quienes ejecutaron estos actos. Como ejemplo citó la ley emitida últimamente en el estado noroccidental de Kaduna en Nigeria, que pide se aplique la castración quirúrgica a los hombres que cometieron violación, la supresión quirúrgica de las trompas de Falopio para el caso de mujeres y pena de muerte en caso la víctima tenga menos de 14 años. A esta legislación se sumaron iniciativas similares en Pakistán, Bangladesh y otros países. (párr. 1-3)

Lo antes descrito genera interrogantes que se resumen al plantear o formular el problema de investigación consistente en ¿Cómo proteger a las víctimas de violencia sexual en el complejo judicial Florida Norte, Guayaquil? 2020?, esta problemática, se ha justificado considerando que hay un incremento de víctimas de violencia sexual, en este sentido, esta justificación se realiza desde una perspectiva teórica, considerando que se logrará afianzar el conocimiento sobre la temática en lo puntual, asimismo porque este trabajo servirá como soporte o base para nuevas investigaciones, es importante resaltar que teóricamente se logrará plantear estrategias de abordaje con el fin de darle un soporte epistemológico a la investigación. En la perspectiva practica se busca aplicar conocimientos que ayuden a encontrar soluciones a los diferentes tipos violencia sexual y la forma en que estos deben ser abordados, recordando que debemos plantear soluciones prácticas que generen espacios de compartir en pos de ampliar la gama de propuestas de solución a los requerimientos de la sociedad. En cuanto a la perspectiva social, podremos viabilizar situaciones en las que la comunidad cuente con un mayor nivel de atención y control de este tipo de violencias y orientar a denunciar a todos aquellos que propicien estos actos delictivos sea en el espacio en que se a necesario. Esto nos permite plantear como

\section{Objetivo general}

- Proponer un Programa de Atención Legal para proteger a las víctimas de violencia sexual en el complejo judicial Florida Norte, Guayaquil. 2020.

\section{Planteando además los siguientes}

\section{Objetivos Específicos}

1. Diagnosticar el estado actual de las víctimas de violencia sexual en el complejo judicial Florida Norte, Guayaquil. 2020. 
2. Identificar los factores influyentes en las víctimas de violencia sexual en el complejo judicial Florida Norte, Guayaquil. 2020,

3. Diseñar Programa de Atención Legal para proteger las víctimas de violencia sexual el complejo judicial Florida Norte, Guayaquil. 2020 y

4. Estimar los resultados que generará la implementación de Programa de Atención Legal en las víctimas de violencia sexual en el complejo judicial Florida Norte, Guayaquil. 2020.

\section{Esto ha permitido platear como}

\section{Hipótesis}

La implementación de un Programa de Atención Legal protegería a las víctimas de violencia sexual en el complejo judicial Florida Norte, Guayaquil. 2020.

Santana (2018) Consecuentemente se realizó un análisis y evaluación de la actual situación donde se da la vulneración por lo que se infiere que en el Código Integral Penal define los derechos de las víctimas, mas no lo hace con las sanciones que se debe aplicar a las personas que realicen la vulneración del derecho a la no re victimización; por lo que, la viabilidad de este estudio es la modificación al Art. 268 donde acuerda la sanción para los que vulneren este derecho. (p. vi)

Devillanova, Fasani y Frattini (2018) Los autores encuentran que los inmigrantes que son potencialmente elegibles para un estatus legal bajo el programa de amnistía tienen una probabilidad significativamente mayor de ser empleados en comparación con los inmigrantes indocumentados que no son elegibles. El tamaño del efecto estimado equivale aproximadamente a la mitad del aumento en el empleo que los inmigrantes indocumentados en nuestra muestra experimentan normalmente durante su primer año en Italia. Estos hallazgos son robustos a varios controles y ejercicios de falsificación. (p. 853)

Malca (2015) Para resguardar a las víctimas del agresión de violación sexual, en la entrevista en Cámara Gessell, nos dice que no sólo bastaría la tecnología implementada en la Cámara, sino que es importante la perito, si la agredida es de sexo femenino y el perito, si el agredido es varón, que va a llevar a cabo la Entrevista Única debiendo ser una profesional competente que ante el agredido conozca las herramientas suficientes para conseguir una prueba idónea e impedir que se victimice, con la finalidad de que no haya 
controversia alguna sobre la calidad de prueba de la entrevista que se realiza en sala de entrevista única en Cámara Gesell. (p. 109)

Flores (2019) en esta investigación se determinó que las víctimas de delitos sexuales de violación, muestran daños y efectos posteriores graves de tipos físicas, psicológicas, sociales, materiales, morales, daño a su plan de vida, daños que totalmente no son analizados, ni alcanzados en su totalidad en las sentencias emitidas porque no se realiza un análisis de cada uno de ellos. (p. 114)

\section{METODOLOGÍA}

\subsection{Tipo y diseño de investigación}

Según el propósito es aplicada. Dado que tiene por finalidad buscar cómo aplicarlo o utilizarlo para el afianzamiento de los saberes adquiridos. Según el enfoque es Mixta, porque se utiliza cuando se requiere una mejor comprensión del problema de investigación, y que no te podría dar cada uno de estos métodos por separado. (QuestionPro, 2021). Según el alcance es Descriptiva, porque muestra los conocimientos de manera que la existencia se da tal y conforme ocurren en un determinado espacio y tiempo dado. Aquí se observa y registra, o también preguntamos y registramos los fenómenos sin generar cambios, debe ser tal como es. (Rojas, 2015) y es Explicativa, porque provocan que algunos hechos expliquen del porqué suceden tales o cuales fenómenos, cuáles son las condiciones y es más porqué se establece la relación entre dos o más variables. (Rusu, s/f). en cuanto al Diseño de investigación este fue no experimental, el cual fue seleccionado por contar con la característica de no manipular las variables en forma intencionada, el fenómeno es observado en su condición natural tal como ocurre, asimismo, la investigación fue de corte transversal, para la recolección los datos en un tiempo y momento únicos, y realizar un analices de su influencia y relación, abarcando grupos o subgrupos, (Hernández-Sampieri, Fernández y Baptista, 2010. p. 120).

\subsection{Variables y operacionalización}

La Variable Independiente Programa de atención legal, es aquella que refiere que la atención legal es la que tiene a cargo brindar la información y asesorar para conseguir solucionar todos los que tengan relación para aplicar normas, reglamentos y leyes en materia de derecho, abarcando todas las variaciones de esta. (párr. 1) 
La Variable Dependiente Las víctimas de violencia sexual, la violencia sexual es cualquier actividad o contacto sexual que ocurre sin su consentimiento. Puede involucrar fuerza física o amenaza de fuerza. También puede ocurrir debido a coerción o amenazas. (MedlinePlus, 2021, párr. 1)

\subsection{Población, muestra y muestreo}

\section{Población}

Arias (2006) precisa que población, es también conocido como conjunto finito o infinito de elementos que guardan una especial relación debido a las características comunes. Esta, queda determinada debido al planteamiento del problema y por el señalamiento del objetivo del estudio. En la investigación objeto de estudio se ha considerado como población a cada uno de abogados y personal técnico del complejo judicial Florida Norte vinculados a temas de violencia sexual, que suman 137 servidores en total

\section{Muestra}

Olivero (2016) Un subgrupo de la población, que se determina bajo ciertos criterios como la representatividad y el número adecuado. Es importante precisar que cuando una población de análisis es relativamente pequeña, a criterio de los investigadores se puede tomar a esa población como la muestra, por lo que debería quedar de la siguiente manera: $\mathrm{N}=\mathrm{n}=137$, Con esa determinación ya no aplican técnicas de muestreo.

\subsection{Técnicas e Instrumentos de recolección de datos}

\section{Técnicas}

Las técnicas que se utilizarán en la investigación están dadas por un conjunto de herramientas e instrumentos que servirán de apoyo para la obtención de datos, (Qualtrics, 2021) refiere que las encuestas son una dinámica de recogida de datos a partir de un muestreo de personas, a menudo con el objetivo de generalizar los resultados para un segmento de población más grande. En el trabajo investigativo se utilizó la encuesta, donde se estableció preguntas de tipo estructuradas relacionadas a la variable de estudio, que permitió la obtención de información. En cuanto a los Instrumentos

Estos vienen hacer el medio que utilizará para guardar la información obtenida, el cuestionario es el cúmulo de preguntas que deben ser respondidas, luego de que han sido estructuradas de la forma más clara posible, de modo que no genere confusión o una mal interpretación. 


\subsection{Procedimientos}

Los procedimientos para la recogida de datos estuvieron dados por la aplicación del instrumento, es decir la encuesta que fue aplicada a abogados y personal técnico del complejo judicial Florida Norte, mediante aplicaciones Web como los formularios de Google drive. Que permitió obtener información sobre las variables de estudios.

\subsection{Método de análisis de datos}

Una vez recolectados los datos a través de las aplicaciones web respectivas, se procedió a la revisión y el análisis minucioso acerca de las respuestas obtenidas, las mismas que se tabularon mediante programas como el excel y el software estadístico SPSS.

\subsection{Aspectos éticos}

Cada proceso investigativo de nivel académico, siempre deben estar sujetos a reglas que pueden determinar qué tan relevantes son los resultados obtenidos:

Respeto, aspecto ético importante dentro de una investigación en la cual se incluyen personas y por lo cual es imperante que se pueden generar situaciones legales o de relación con la moral, en tal sentido se consideran los derechos de quienes son considerados dentro de la investigación.

Transparencia, referido a la conducta humana, lo cual permite que todos comprendan de la forma más fácil el mensaje de lo que se quiere expresar, teniendo en consideración que ser transparente es ser claro, a tal manera de no dejar que se den malas interpretaciones.

Confiabilidad, referido a la capacidad de lograr realizar una función específica y que esta conlleve a generar confianza sobre los resultados hallados a través de procesos considerados adecuados y permitidos.

Autonomía ejecutar un estudio investigativo, debe asegurarse de la independencia que el equipo investigador debe tener, pues los hallazgos a los que se arriben tendrán una connotación en la sociedad. 


\section{RESULTADOS Y DISCUSIÓN}

\section{Tabla 1}

Las directrices legales están dando soporte al programa de atención o asesoría legal

\begin{tabular}{ccc}
\hline Descripción & $\mathbf{f i}$ & $\mathbf{\%}$ \\
\hline TD & 37 & 27.00 \\
D & 54 & 39.42 \\
NO & 28 & 20.44 \\
A & 18 & 13.14 \\
\hline Total & $\mathbf{1 3 7}$ & $\mathbf{1 0 0 . 0 0}$ \\
\hline
\end{tabular}

\section{Fuente: El Autor}

Conforme a los datos conseguidos en la Tabla 1, sobre si Las directrices legales están dando soporte al programa de atención o asesoría legal, al respecto un $27 \%$ manifiestan estar Totalmente en Desacuerdo y un $39.42 \%$ solo refieren estar en Desacuerdo con tal afirmación, asimismo un $20.44 \%$ tienen dudas y prefieren No Opinar, mientras que un $13.14 \%$ ante dicha afirmación refieren estar de Acuerdo. Estos resultados se corroboran con lo manifestado por Pineda, González y Fernández. (2021) quienes precisan la imperiosa necesidad de contar con una ley o normas que puedan regular el accionar por parte del estado en función a la imperante necesidad de prestar el necesario, dado que sin ello la población que está expuesta no se sentirá respaldad por sus autoridades o por el gobierno que los dirige. En esa misma idea Strassle y Berkman (2020) muestran que hay regulaciones y sobre todo en materia de protección que han sido anuladas y con ello se puede evidenciar el desamparo en el que se siente la población.

\section{Tabla 2}

La atención terapéutica viene ayudando psicológicamente a la población víctima de violencias

\begin{tabular}{ccc}
\hline Descripción & $\mathbf{f i}$ & $\boldsymbol{\%}$ \\
\hline TD & 51 & 37.22 \\
D & 22 & 16.06 \\
NO & 52 & 37.96 \\
A & 12 & 8.76 \\
\hline Total & $\mathbf{1 3 7}$ & $\mathbf{1 0 0 . 0 0}$ \\
\hline
\end{tabular}

Fuente: El Autor

Asimismo, conforme a los datos conseguidos en la Tabla 2, sobre si La atención terapéutica viene ayudando psicológicamente a la población víctima de violencias, al respecto un $37.22 \%$ manifiestan estar Totalmente en Desacuerdo y un $16.06 \%$ solo 
refieren estar en Desacuerdo con tal afirmación, asimismo un $37.96 \%$ tienen dudas y prefieren No Opinar, mientras que un $8.76 \%$ ante dicha afirmación refieren estar de Acuerdo. Dichos resultados evidencian lo manifestado por Jaramillo-Sierra y RipollNúñez (2018) quienes puntualizaron que no se está obteniendo la sensibilización que se busca a través de los programas legales, el de brindar ayuda o soporte terapéutico para las personas que se ven afectados por algún tipo de violencia o daño que se causa, aún cuando lo deseado sería eliminar la violencia física, la reducción de la violencia psicológica y el incremento del tiempo compartido, el conversatorio y la intimidad de la pareja. Por último, se lleva a discusión los hallazgos referidos a otros programas respaldados en evidencia para violencia de pareja. (p. 55)

\section{Tabla 3}

La reducción de riesgos personales viene siendo la consecuencia de aplicar una cultura de prevención

\begin{tabular}{ccc}
\hline Descripción & fi & \% \\
\hline D & 26 & 18.98 \\
NO & 22 & 16.06 \\
A & 28 & 20.44 \\
TA & 61 & 44.52 \\
\hline Total & $\mathbf{1 3 7}$ & $\mathbf{1 0 0 . 0 0}$ \\
\hline
\end{tabular}

\section{Fuente: El Autor}

Por otro lado conforme a los datos conseguidos en la tabla 3, sobre si La reducción de riesgos personales viene siendo la consecuencia de aplicar una cultura de prevención, al respecto un $18.98 \%$ solo refieren estar en Desacuerdo con tal afirmación, asimismo un $16.06 \%$ tienen dudas y prefieren No Opinar, mientras que un $20.44 \%$ ante dicha afirmación refieren estar de Acuerdo y finalmente el $44.52 \%$ están Totalmente de Acuerdo con lo manifestado. Estos resultados se corroboran con lo manifestado por Rzhevskaya et al. (2020) quienes precisan que se requiere desarrollar actos legales regulatorios que controlen el procedimiento de restricción de los derechos de los individuos con trastornos mentales y criterios médicos. El enfoque bioético a la prestación de atención psiquiátrica, el uso de programas psicoeducativos y la rehabilitación psicosocial promueven el cumplimiento de los pacientes y una alianza terapéutica efectiva con un psiquiatra. (p. 568)

\section{Tabla 4}

El daño físico que sufren las víctimas de violencia sexual cada día se evidencia como lesiones graves 


\begin{tabular}{ccc}
\hline Descripción & $\mathbf{f i}$ & \% \\
\hline D & 17 & 12.41 \\
NO & 29 & 21.17 \\
A & 59 & 43.07 \\
TA & 32 & 23.35 \\
\hline Total & $\mathbf{1 3 7}$ & $\mathbf{1 0 0 . 0 0}$ \\
\hline
\end{tabular}

Fuente: El Autor

Conforme a los datos conseguidos en la Tabla 4, sobre si El daño físico que sufren las víctimas de violencia sexual cada día se evidencian como lesiones graves, al respecto un $12.41 \%$ solo refieren estar en Desacuerdo con tal afirmación, asimismo un $21.17 \%$ tienen dudas y prefieren No Opinar, mientras que un $43.07 \%$ ante dicha afirmación refieren estar de Acuerdo y finalmente el $23.35 \%$ están Totalmente de Acuerdo con lo manifestado. Los resultados mostrados corroboran lo hallado pues, lo manifestado por Malca (2015) sugiere Para resguardar a las víctimas del agresión de violación sexual, en la entrevista en Cámara Gessell, nos dice que no sólo bastaría la tecnología implementada en la Cámara, sino que es importante la perito, si la agredida es de sexo femenino y el perito, si el agredido es varón, que va a llevar a cabo la Entrevista Única debiendo ser una profesional competente que ante el agredido conozca las herramientas suficientes para conseguir una prueba idónea e impedir que se victimice, con la finalidad de que no haya controversia alguna sobre la calidad de prueba de la entrevista que se realiza en sala de entrevista única en Cámara Gesell. (p. 109)

\section{CONCLUSIONES}

1. La presente investigación concluye con una propuesta sobre la implementación de un Programa de Atención Legal para proteger a las Víctimas de Violencia Sexual en el Complejo Judicial Florida Norte, Guayaquil. 2020, programa que es muy necesario, pues la problemática referida a las víctimas de violencia sexual está aumentando y necesita ser abordada desde los diferentes estamentos del gobierno y con ello generar confianza en aquellas personas que fueron víctimas de este tipo de violencia.

2. Se identificaron aspectos muy notorios que tienen a las víctimas de violencia sexual, las lesiones son de diferentes tipos y magnitudes, incluso llegando a la muerte, una inestabilidad, nerviosismo sumado al trauma y temor permanente. 
3. Se analizaron factores saltantes que son los que se asocian directamente a la inestabilidad de las víctimas de violencia sexual como daños físicos que cada vez tienen manifestaciones de lesiones fuertes, psicológicos y su prevalencia en la nueva conducta que asume la víctima y morales frente a no saber manejar la llamada deshonra que sufrió.

4. Se elaboró un programa con un diseño que aborde en enfoque integral de acción, para lo cual se consideró la parte legal, el tiempo de la atención terapéutica y como desarrollar una cultura preventiva, con lo cual se pretende reducir los niveles de inseguridad y desprotección en que hoy se sienten las víctimas de violencia sexual.

5. Los resultados que se obtuvieron, a través del juicio de expertos, estadísticamente demuestran la importancia y necesidad de aplicar este programa, revisada y evaluada por especialistas, conocedores de la gestión del estado y con el pertinente aporte de legalidad de intervención, arrojan un Coeficiente de validez de contenido es 0.916 con lo cual se determina la pertinencia de implementar y aplicar la estrategia

\section{REFERENCIAS BIBLIOGRAFICAS}

Alizadeh, S., Riazi, H., Majd, H. A. y Ozgoli, G. (2021). The effect of sexual health education on sexual activity, sexual quality of life, and sexual violence in pregnancy: a prospective randomized controlled trial. BMC Pregnancy \& Childbirth, 21(1), 1-11. https://doi.org/10.1186/s12884-021-03803-8

Alkan, Ö. Y Tekmanlı, H. (2021). Determination of the factors affecting sexual violence against women in Turkey: a population-based analysis. BMC Women's Health, 21(1), 1-15. https://doi.org/10.1186/s12905-021-01333-1

Ballesteros, M. y García, M. (2018). Los programas de cumplimiento penal: origen, regulación, contenido y eficacia en el proceso. Anuario Jurídico y Económico Escurialense,

197-221. https://dialnet.unirioja.es/servlet/articulo?codigo $=6332784$

Barrera, A., Martínez, ,J. y Vásquez, E. (2017). Modelo de gestión para la aplicación del control metrológico legal y la evaluación de la conformidad en equipos biomédicos. Revista Ingeniería Biomédica, 11(21), 65-72. http://dx.doi.org/10.24050/19099762.n21.2017.1175

Barrera, A., Martínez, J. y Vásquez, E. (2017). Modelo de gestión para la aplicación del control metrológico legal y la evaluación de la conformidad en equipos 
biomédicos. Revista Ingeniería Biomédica, 11(21), 65-72. http://dx.doi.org/10.24050/19099762.n21.2017.1175

Bembibre, C. (2010). Definición de Legalidad. Definición ABC. https://bit.ly/3183YG1

Boxall, H. y Morgan, A. (2021). Who is most at risk of physical and sexual partner violence and coercive control during the COVID-19 pandemic? Trends \& Issues in Crime \& Criminal Justice, 618, 1-19. https://doi.org/10.52922/ti78047

Bustos, R. y Pastrana, E. (2018). «Good Training for Good Judgments». El Programa Help (Human Rights Education for Legal Professionals) De Formación en Derechos Humanos Del Consejo De Europa. Teoria y Realidad Constitucional, 2019(42), 527-538. http://revistas.uned.es/index.php/TRC/article/view/23647

Cavagnoud R. (2018). Violencia contra las mujeres en el Perú. ¿Cuál es el alcance del movimiento "Me Too" en el acceso al apoyo constitucional?. Párr. 1-4. https://ifea.hypotheses.org/1283

Centro de Noticias OMS (Organización Mundial de la Salud). (2020). Violencia juvenil. párr. 6-11 Centro de prensa. https://www.who.int/es/news-room/factsheets/detail/youth-violence

Champion, A., Oswald, F. y Pedersen, C. L. (2021). Technology-facilitated sexual violence and suicide risk: A serial mediation model investigating bullying, depression, perceived burdensomeness, and thwarted belongingness. Canadian Journal of Human Sexuality, 30(1), 125-141. https://doi.org/10.3138/cjhs.20200044

Chang, G. (2018). Breves apuntes sobre el daño moral: la apuesta por su presunción e intentos de cuantificación. Pasión por el Derecho. https://bit.ly/2OpLJ2W

Chiang, M., Crispi, F. y Carrillo, A. (2020). Unidad Clínico Forense del Hospital Gustavo Fricke: Modelo experimental de la Norma Técnica Nacional de Atención a Víctimas de Violencia Sexual. Cuad Méd Soc (Chile). 3, pp. 13 - 19. https://bit.ly/3wVEosv

Chugani, C., Anderson, J., Richter, R., Bonomi, A., DeGenna, N., Feinstein, Z., Jones, K. y Miller, E. (2021). Perceptions of College Campus Alcohol and Sexual Violence Prevention among Students with Disabilities: "it Was a Joke." Journal of Family Violence, 36(3), 281-291. https://doi.org/10.1007/s10896-020-00150-8 
Conamed (2014). Daño Físico diccionario.leyderecho.org https://diccionario.leyderecho.org/dano-fisico/

Corbin, J. (2021). Los 13 tipos de agresión sexual (características y efectos). Sicologia y Mente. https://psicologiaymente.com/forense/tipos-de-agresion-sexual

Dame, J., Oliffe, J., Hill, N., Carrier, L. y Evans-Amalu, K. (2020). Sexual violence among men who have sex with men and two-spirit peoples: A scoping review. Canadian Journal of Human Sexuality, 29(2), 240-248. https://doi.org/10.3138/cjhs.2020-0014

De La Hermosa, M. y Polo, C. (2018). Sexualidad, violencia sexual y salud mental. Revista de La Asociación Española de Neuropsiquiatría, 38(134), 349-356. https://scielo.isciii.es/pdf/neuropsiq/v38n134/2340-2733-raen-38-134-0349.pdf

de Villiers, T., Duma, S. y Abrahams, N. (2021). "As young men we have a role to play in preventing sexual violence": Development and relevance of the men with conscience intervention to prevent sexual violence. PLoS ONE, 16(1), 1-16. https://doi.org/10.1371/journal.pone.0244550

Devillanova, C., Fasani, F. y Frattini, T. (2018). Employment of Undocumented Immigrants and the Prospect of Legal Status: Evidence from an Amnesty $\begin{array}{llll}\text { Program. } & \text { ILR } & \text { 853-881(4), }\end{array}$ https://doi.org/10.1177/0019793917743246

Espinoza, C., Lima, Y., Valle, C., Nuñez, M., Calderón, A., Hidalgo, T., Guzmán, J. y Carrión, E. (2019). Transfusión sanguínea en la práctica clínica e implicaciones ético-legales. Archivos Venezolanos De Farmacologia y Terapéutica, 38(6), $797-$ 803. https://bit.ly/3wUy9Fi

Duran, R. y Vergara, A. (2008). El mejoramiento de vivienda y entorno en bogotá 19941998: Programas, conceptos y estrategias. Investigación \& Desarrollo, 16(1). https://bit.ly/3gbrVKq

Educo (2017). Maltrato psicológico: ¿cómo afecta a los niños? https://bit.ly/3tbeRcU El Centro Nacional de Recursos contra la Violencia Sexual (2012). ¿Qué es la Violencia Sexual?. Información General. https://www.nsvrc.org/sites/default/files/Publications_NSVRC_Overview_Quees-la-Violencia-Sexual.pdf 
Escobar, C., Rodríguez, N. y Rojas, F. (2017). Estrategia para mejorar la práctica jurídica desarrollada en el Consultorio Jurídico. [Strategy to improve the legal practice developed in the Legal Office]. Revista Logos, Ciencia \& Tecnologia, 9(2), 97111. https://doi.org/10.22335/rlct.v9i2.454

Giraldo, L. y Muñoz, E. (2020). La violencia sexual en el conflicto armado en tiempos de transición: el caso del departamento de Caldas. Revista Juridicas, 17(2), 159-179. https://doi.org/10.17151/jurid.2020.17.2.9

Flores, A. (2019). La reparación integral de las víctimas en los delitos sexuales de violación en el Ecuador. Universidad Central del Ecuador. pp. 1 -141. http://www.dspace.uce.edu.ec/handle/25000/19008

Fonzo, C. (2020). Sensibilidades legales y usos alternativos del derecho. El encierro ganadero comunitario "El Rejunte" (Figueroa, Santiago del Estero). Cuestiones De Sociología, (23)http://dx.doi.org/10.24215/23468904e106

Galaz, C., Guarderas, P. (2017). La intervención psicosocial y la construcción de las "mujeres víctimas". Una aproximación desde las experiencias de Quito (Ecuador)

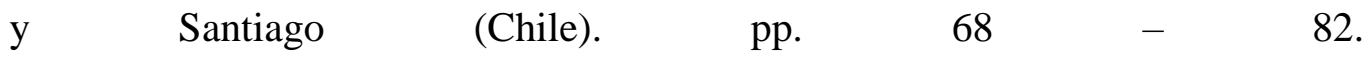
https://dialnet.unirioja.es/servlet/articulo?codigo=5908026

Giammattei A. (2019) Consecuencias Que Se Derivan De La Violencia Sexual. Secretaria contra la Violencia Sexual, Explotación y trata de Personas. Gobierno de Guatemala. párr. 1-5. https://www.svet.gob.gt/temasdetrabajo/consecuenciasque-se-derivan-de-la-violencia-sexual

Guzmán Sarmiento, D. C., \& Julián Ignacio Ordoñez Losada. (2020). Fundamentos constitucionales, legales y jurisprudenciales de la restitución de tierras en Colombia. Revista Jurídica Piélagus, 19(2)http://dx.doi.org/10.25054/16576799.2872

Heathwhise (2020). Maltrato físico. https://www.cigna.com/es-us/individualsfamilies/health-wellness/hw/temas-de-salud/maltrato-fsico-viobh

Holt K. (2020). Bachelet: La pena de muerte o la castración no son respuestas adecuadas ante una violación. Noticias ONU. Párr. 1-3. https://news.un.org/es/story/2020/10/1482452 
Howard, R. M., Potter, S. J., Guedj, C. E., \& Moynihan, M. M. (2019). Sexual violence victimization among community college students. Journal of American College Health, 67(7), 674-687. https://doi.org/10.1080/07448481.2018.1500474

Jaramillo-Sierra, A., \& Ripoll-Núñez, K. (2018). Adaptación de un programa de intervención para violencia situacional de parejas *. Revista De Estudios Sociales, (66), 55-70. http://dx.doi.org/10.7440/res66.2018.06

Johnson, K. M., Lederer, A. M., Liddell, J. L., Sheffield, S., \& McCraw, A. (2021). Teaching to Impact Sexual Violence? The Evaluation of a Curricular Intervention for First-Year College Students. American Journal of Health Promotion, 35(3), 438-441. https://doi.org/10.1177/0890117120967604

Lamas, M. (2016). Concepto de Daño Psíquico: la Historia y su Evolución Conceptual. Ministerio Público. Lima - Perú. Pág. 1 - 93. https://bit.ly/38Ay2Fb

Leeuw, F. L. (2017). American Legal Realism: Research Programme and Policy Impact. Utrecht Law Review, 13(3), 28-40. https://doi.org/10.18352/ulr.412

Malca, E. (2015). Protección a víctimas del abuso sexual. Universidad Privada Antenor Orrego. pp. 1 - 124. https://repositorio.upao.edu.pe/handle/20.500.12759/973

Maldonado, J. (2019). Violencia política sexual: una conceptualización necesaria. Nomadías, 27, 143-166. DOI: 10.5354/0719-0905.2019.54379

Manzo, M. (2015). Daño Psíquico. Definición y evaluación. Sus dificultades. NOBLE Compañía de Seguros. https://bit.ly/3rHEpxP

Martinez, M. (2017). La Cultura Preventiva. https://bit.ly/3bDqd3o

McCray, K., \& Taylor, E. (2021). "I learned that sports teaches rape culture": Assessing Sexual Violence Prevention Education for Intercollegiate Athletes. Journal of Intercollegiate Sport, 14(1), 46-65. https://doi.org/10.17161/jis.v14i1.13428

MedlinePlus (2021). Violencia sexual. Biblioteca Nacional de Medicina de los EE. UU. https://bit.ly/2OLHhLI

Ministerio de Trabajo, Migraciones y Seguridad Social (2019). Cultura Preventiva. https://bit.ly/3bH56NL

Mis Abogados (2017). ¿Qué es la violencia física? https://bit.ly/3cr3IxL

Morán Garreta, L., Pinzón Gómez, E., Muñoz Villa, V., Lesmes Duque, M. y Victoria García, M. (2017). Abuso Sexual en Mujeres De 10 a 13 Años en El Valle Del 
Cauca, Colombia. Archivos de Medicina (1657-320X), 17(1), 121-130. https://doi.org/10.30554/archmed.17.1.1913.2017

Mueller, V., Billings, L., Mogues, T., Peterman, A. y Wineman, A. (2018). Filling the legal void? Impacts of a community-based legal aid program on women's landrelated knowledge, attitudes, and practices. Oxford Development Studies, 46(4), 453-469. https://doi.org/10.1080/13600818.2017.1414174

Mwanukuzi, C., \& Nyamhanga, T. (2021). "It is painful and unpleasant": experiences of sexual violence among married adolescent girls in Shinyanga, Tanzania. Reproductive Health, 18(1), 1-7. https://doi.org/10.1186/s12978-020-01058-8

Navarro, P. (s/f). ¿De qué hablamos cuando hablamos de Cultura Preventiva? https://bit.ly/38vZ3te

Nelon, J., De Pedro, K., Gilreath, T., Patterson, M., Holden, C. y Esquivel, C. (2019). A latent class analysis of the co-occurrence of sexual violence, substance use, and mental health in youth. Substance Use \& Misuse, 54(12), 1938-1944. https://doi.org/10.1080/10826084.2019.1618337

OEA (s/f). Descripción, Funciones Y Objetivos. Departamento de Asesoría legal. https://bit.ly/3vh2sGh

Oyarce, J. (2018). Libertad Y Proyecto De Vida De Los Adolescentes Víctimas De Abuso Sexual. pp. 93 - 106. https://dialnet.unirioja.es/servlet/articulo?codigo=6523163

Paredes-Mosquera, H., Guachetá-Torres, J., y Paredes-Londoño, E. (2018). Las víctimas de violencia sexual en el marco del conflicto armado en relación con los procesos de paz en Colombia, 1991 a 2017. Revista Jurídicas, 15 (1), pp. 88 -109. DOI: https://doi.org/10.17151/jurid.2018.15.1.6.

Pérez, J., Merino, M. (2014). Definición de Terapéutico. https://bit.ly/3evxa8x

Pérez, T. (2018). Conoce y Domina las Habilidades Terapéuticas fundamentales del Psicólogo. Psiky. https://bit.ly/3tdgyqc

Pineda Folgoso, L., González Hernández, A., \& Fernández López, L. A. (2021). La atención médica y legal en la especialidad de Ortopedia y Traumatología. Revista Cubana de Ortopedia y Traumatología, 35(1), 1-6. http://www.revortopedia.sld.cu/index.php/revortopedia/article/view/374

Ramos, A. (2017). Influencia De Los Contextos Familiares Disfuncionales En La Comisión De Delitos Sexuales Intrafamiliares En La Región Tacna, 2012-2014. 
Universidad Privada De Tacna. $\quad$ Pp. 1 - 256. http://repositorio.upt.edu.pe/handle/UPT/381

Rátiva-Velandia, M. (2018). Escuela Normal colombiana frente a las disposiciones legales. Tensiones pedagógicas y administrativas. Praxis, 14(1), 9-24. http://dx.doi.org/10.21676/23897856.2537

Ríos, J., \& Brocate, R. (2017). Violencia sexual como crimen de lesa humanidad: los casos de Guatemala y Perú. Revista CIDOB d'Afers Internacionals, 117, 79-99. https://doi.org/10.24241/rcai.2017.117.3.79

Romero, C. (2015). “La Reparación Integral A Las Víctimas De Delitos Sexuales Con Fundamento En El Derecho A Una Vida Digna". Pp. 1 -219. https://repositorio.pucesa.edu.ec/handle/123456789/1233

Roser, F. Flórez, J. La atención: bases fundamentales. https://bit.ly/3qHi2rc

Rzhevskaya, N. K., Ruzhenkov, V. A., Ruzhenkova, V. V., Khamskaya, I. S., \& Moskvitina, U. S. (2020). Psychiatric coercion and violence: ethical, legal and preventive aspects. Archivos Venezolanos De Farmacologia y Terapéutica, 39(5), 568-572. http://dx.doi.org/10.5281/zenodo.4265434

Sage (s/f). Asesoría Jurídica. Términos del Diccionario Empresarial. https://bit.ly/3thwIz6

Salazar, D. (2019). Abuso y violencia sexual en mujeres en la ciudad de Guayaquil. Universidad Politécnica Salesiana Sede Guayaquil. pp. 1 - 32. https://dspace.ups.edu.ec/handle/123456789/18063

Sandoval, Y. V. (2020). Seguridad social en pensiones desde la perspectiva de género: un acercamiento constitucional y legal de Chile, Brasil y Colombia *. Hallazgos, 17(33), 53-77. http://dx.doi.org/10.15332/2422409X.5225

Sangma, K. y Subramaniyan, J. (2021). Sexual Violence against Women in Meghalaya. Ilkogretim Online, 20(4), 1470-1477. https://doi.org/10.17051/ilkonline.2021.04.169

Santana, K. (2018). Vulneración Del Derecho Constitucional A La No revictimización De Los Niños Y Niñas Víctimas De Delitos Sexuales, En Su Paso Por El Sistema Procesal Penal En El Cantón Santa Elena, Desde Agosto Del 2014 Hasta Diciembre Del 2016. Universidad Católica De Santiago De Guayaquil. pp. 1 58. http://repositorio.ucsg.edu.ec/handle/3317/10832 
Significados (2017). Significado de Legalidad. https://bit.ly/2OMfFpA

Silva, M. (2015). Características de la Cultura Preventiva. https://bit.ly/38wmra6

Spence, C., Bachmeier, J. D., Altman, C. E., \& Hamilton, C. (2020). The Association Between Legal Status and Poverty Among Immigrants: A Methodological Caution. Demography (Springer Nature), 57(6), 2327-2335. https://doi.org/10.1007/s13524-020-00933-0

STRASSLE, C., \& BERKMAN, B. E. (2020). Workplace Wellness Programs: Empirical Doubt, Legal Ambiguity, and Conceptual Confusion. William \& Mary Law Review, 61(6), 1663-1717. https://bit.ly/3vSdh1o

Tamayo, M., Tamayo, L., Tamayo, E. (2019). La violencia se vive de miles maneras: Voces de mujeres víctimas de violencia sexual en el conflicto armado del Carmen de Bolívar - Región Caribe, Colombia, 2018-2019*. pp. 9 - 34. https://www.redalyc.org/articulo.oa?id=31662848001

Thomas, A. J. (2018). Secure Choice: State-Based Retirement Savings Programs Move Forward Despite Regulatory and Legal Headwinds. Benefits Magazine, 55(10), 52-58. https://www.ifebp.org/inforequest/ifebp/0201147.pdf

UNAM (2014). DAÑO MORAL Y SU REPARACIÓN. Universidad Nacional Autónoma de México. Pág. 51 - 120. https://bit.ly/2PXiDbE

UNHCR - ACNUR. (2019). La Agencia de la ONU para los Refugiados. Boletín. párr. 1. https://www.acnur.org/violencia-sexual-y-degenero.html?gclid=EAIaIQobChMIoLOIzIK47QIV6suGCh0MoghIEAMYASA AEgK_J_D_BwE

VanSingel, A. J. (2019). The Calm After the Storm: 45 Years of the Aba Young Lawyers Division's Disaster Legal Services Program. Touro Law Review, 35(3), 10191086. https://core.ac.uk/download/pdf/270297251.pdf 